\title{
Are n-3 Polyunsaturated Fatty Acids Antiarrhythmic in the Absence of Ischemia?
}

\author{
Editorial to: "The Role of $n$-3 PUFAs in Preventing the Arrhythmic Risk in Patients \\ with Idiopathic Dilated Cardiomyopathy" by S. Nodari et al.
}

Meritt H. Raitt

Published online: 13 November 2008

(C) Springer Science + Business Media, LLC 2009

In this issue of Cardiovascular Drugs and Therapy Nodari et al. [1] add to the growing fund of knowledge on the possible antiarrhythmic effects of $n-3$ polyunsaturated fatty acids ( $n-3$ PUFA). In this relatively small study the investigators found that, compared to placebo, 6 months of supplementation with $n$-3 PUFA improved a wide range of markers of the risk of ventricular arrhythmias and sudden death in patients with non-ischemic cardiomyopathy and frequent ventricular ectopy. These observations included improved findings on microvolt $\mathrm{T}$-wave analysis, QRS signal averaging, non sustained arrhythmias on Holter, heart rate variability analysis, catecholamine and cytokine plasma levels, left ventricular function, and exercise capacity. The breadth of these potentially beneficial effects is remarkable. Perhaps more remarkable is that these effects were observed in patients with non-ischemic cardiomyopathy. As I will discuss below, to date the antiarrhythmic effects of $n-3$ PUFA have been demonstrated primarily in ischemic ventricular fibrillation animal models and in patients with coronary artery disease at risk for acute ischemia.

The idea that $n$-3 PUFA might protect against death from heart disease dates back to the observation of a lower than expected risk of coronary artery disease in Greenland Eskimos who have a diet that is especially high in $n-3$ PUFA [2]. Subsequent observational studies have shown that a diet high in fish especially cold water fatty fish which

\footnotetext{
M. H. Raitt ( $₫)$

Portland VA Medical Center,

Portland, OR, USA

e-mail: merritt.raitt@va.gov

M. H. Raitt

P-3-CARD,

3710 SW US Veterans Road,

Portland, OR 97239, USA
}

are rich in $n$-3 PUFA is associated with a reduced risk of death from heart disease. The concept that $n$-3 PUFA may reduce cardiac deaths via antiarrhythmic effects is supported by basic science, animal, and human studies. In vitro studies have shown that acutely superfused $n-3$ PUFA can interact with sodium [3], potassium [4] and calcium ion channels $[5,6]$ in the cell membrane. Of these interactions the most important appears to be the ability of $n-3$ PUFA to inactivate sodium channels in the presence of myocardial ischemia [3]. Rats chronically fed a diet high in $n-3$ PUFA were significantly less likely to have ventricular fibrillation during acute myocardial ischemia than were animals fed a control diet [7]. Similarly, the acute infusion of $n-3$ PUFA in a dog model of acute ischemic ventricular fibrillation was associated with a marked reduction in the risk of the arrhythmia [8]. The Physician's Health study showed a reduction in sudden death with higher fish intake but no reduction in the risk of myocardial infarction [9]. When fatty acids levels were examined in this population there was a strong inverse relationship between $n$-3 PUFA levels but not other fatty acids and the risk of sudden death [10]. Similarly, a case control study that compared patients who had suffered a cardiac arrest and matched controls found significantly higher fish intake and $n-3$ PUFA levels in controls [11].

The most convincing evidence of an antiarrhythmic effect of $n$-3 PUFA comes from the GISSI investigators [12] who entered 11,324 patients with a recent myocardial infarction into a study in which subjects were randomized to $300 \mathrm{mg}$ of vitamin E, $850 \mathrm{mg}$ of $n-3$ PUFA, both vitamin E and $n-3$ PUFA, or neither. Patients assigned to $n-3$ PUFA had a $20 \%$ reduction in all cause mortality and a $45 \%$ reduction in the risk of sudden death. Vitamin E showed no benefit. This significant reduction in sudden death has been interpreted as a strong indicator that the benefit was 
primarily antiarrhythmic. It has not gone unnoticed that the best evidence for the antiarrhythmic effects of $n$ - 3 PUFA is in animal models of ischemic ventricular fibrillation and in human patients with recent myocardial infarction. The most widely accepted explanation for this beneficial effect is that the effect of $n-3$ PUFA on sodium channels is most prominent during ischemia and thus the antiarrhythmic effects of $n-3$ PUFA will be most prominent in ischemic arrhythmias such as would be expected to occur in post myocardial infarction patients like those enrolled in the GISSI trial.

Indirect evidence that ischemia may be a prerequisite for antiarrhythmic effects of $n-3$ PUFA comes from studies in which patients with implantable defibrillator were randomized to $n-3$ PUFA or control [13-15]. These studies enrolled patients who all had prior documented ventricular arrhythmias outside the setting of acute myocardial infarction. Such arrhythmias are likely in most cases due to reentry facilitated by slow conduction in and around areas of myocardial scar related to infarction or cardiomyopathy. A meta analysis of these studies showed no antiarrhythmic effect of $n-3$ PUFA in this population [16].

Given the evidence to date that the antiarrhythmic benefit of $n$-3 PUFA is most prominent in acute ischemia the findings of Nodari et al. are potentially quite important. As the authors point, out there have not been large studies of $n-3$ PUFA in patients with non-ischemic cardiomyopathy. Given the prevailing wisdom that ischemia is an important prerequisite for the antiarrhythmic effects of $n-3$ PUFA the remarkable breadth of the potentially beneficial effects of $n-3$ PUFA in the study population is remarkable. Though the study was well designed there are aspects of it that may call into question the broad application of the findings. The first issue is that $93 \%$ of the patients were on amiodarone. Could there be an interaction between amiodarone and $n-3$ PUFA such that these findings may not be seen in patients not taking amiodarone? Second only patients with frequent ectopy were eligible for the study. Could the reduction in the density of premature beats in these patients with frequent ectopy at baseline have indirectly led to some of the other observed changes though revere remodeling and improved hemodynamics [17]? Would the same spectrum of affects been seen in patients without frequent premature ventricular contractions at baseline?

Finally, one has to be very careful about extrapolating from improvements in markers for the risk of ventricular arrhythmias to the actual prevention of arrhythmias. The authors cite the classic example of this phenomenon, the Cardiac Arrhythmia Suppression Trial (CAST) [18]. Frequent premature ventricular contractions are a well know risk for an increased risk severe ventricular arrhythmias in patients with prior myocardial infarction. In CAST when patients with prior myocardial infarction in whom pre enrollment testing had show flecainide to suppress these premature beats were randomized to flecainide or placebo the sudden death rate was significantly higher in patients randomized to flecainide compared to placebo. This important lesson, that improvement in markers of risk may not translate to improvement in outcome and may even be associated with worse outcome, is an important lesson that cardiology and all of medicine should never forget. In this light, it is interesting to note that there was a trend toward a reduced risk of ventricular arrhythmias in the one implantable defibrillator study that specifically reported results in patients without coronary artery disease [13]. The work by Nodari et al. [1] is an excellent first step but larger prospective trials of $n-3$ PUFA with mortality or documented severe arrhythmias as endpoints need to be performed before $n$-3 PUFA supplementation can be routinely recommended for the prevention of sudden death in patients with non-ischemic cardiomyopathy.

\section{References}

1. Nodari S, Metra M, Milesi G, Manerba A, Cesana B, Gheorghiade $\mathrm{M}$, et al. The role of $n-3$ PUFAs in preventing the arrhythmic risk in patients with idiopathic dilated cardiomyopathy. Cardiovasc Drugs Ther. 2009;23:5-15.

2. Bang HO, Dyerberg J, Nielsen A. Plasma lipid and lipoprotein pattern in greenlandic west-coast Eskimos. Lancet 1971;297: 1143-6.

3. Xiao YF, Kang JX, Morgan JP, Leaf A. Blocking effects of polyunsaturated fatty acids on $\mathrm{Na}+$ channels of neonatal rate ventricular myocytes. Physiology (Bethesda) 1995;92:11000-4.

4. Honore E, Barhanin J, Attali B, Lesage F, Lazdunski M. External blockade of the major cardiac delayed-rectifier $\mathrm{K}+$ channel (Kv1.5) by polyunsaturated fatty acids. Proc Natl Acad Sci U S A 1994;91:1937-44.

5. Hazama H, Nakajima T, Asano M, et al. Omega-3 polyunsaturated fatty acids-modulation of voltage-dependent L-type Ca2+ current in guinea-pig tracheal smooth muscle cells. Eur J Pharmacol 1998;355:257-66.

6. Danthi S, Enyeart J, Enyeart J. Modulation of native T-type calcium channels by omega-3 fatty acids. Biochem Biophys Res Commun 2005;327:485-93.

7. McLennan PL, Bridle TM, Abeywardena MY, Charnock JS. Comparative efficacy of $n-3$ and $n-6$ polyunsaturated fatty acids in modulating ventricular fibrillation threshold in marmoset monkeys. Am J Clin Nutr 1993;58:666-9.

8. Billman GE, Kang JX, Leaf A. Prevention of sudden cardiac death by dietary pure omega-3 polyunsaturated fatty acids in dogs. Circulation 1999;99:2452-7.

9. Albert CM, Hennekens CH, O'Donnell CJ, Ajani UA, Carey VJ, Willett WC, et al. Fish consumption and risk of sudden cardiac death. JAMA 1998;279:23-8.

10. Albert CM, Campos H, Stampfer MJ, et al. Blood levels of longchain $n-3$ fatty acids and the risk of sudden death. N Engl J Med 2002;346:1113-8.

11. Siscovick DS, Raghunathan TE, King I, et al. Dietary intake and cell membrane levels of long-chain $n$-3 polyunsaturated fatty acids and the risk of primary cardiac arrest. JAMA 1995;274:1363-7. 
12. Marchioli R, Barzi F, Bomba E, et al. Early protection against sudden death by $n-3$ polyunsaturated fatty acids after myocardial infarction: time-course analysis of the results of the Gruppo Italiano per lo Studio della Sopravvivenza nell'Infarto Miocardico (GISSI)-Prevenzione. Circulation 2002;105:1897-903.

13. Raitt MH, Connor WE, Morris C, et al. Fish oil supplementation and risk of ventricular tachycardia and ventricular fibrillation in patients with implantable defibrillators: a randomized controlled trial. JAMA 2005;293:2884-91.

14. Leaf A, Albert CM, Josephson M, et al. Prevention of fatal arrhythmias in high-risk subjects by fish oil $n-3$ fatty acid intake. Circulation 2005;112:2762-8.

15. Brouwer IA, Zock PL, Camm AJ, et al. Effect of fish oil on ventricular tachyarrhythmia and death in patients with implantable cardioverter defibrillators: the Study on Omega-3 Fatty Acids and Ventricular Arrhythmia (SOFA) randomized trial. JAMA 2006;295:2613-9.

16. Jenkins DJA, Josse AR, Beyene J, et al. Fish-oil supplementation in patients with implantable cardioverter defibrillators: a metaanalysis. CMAJ 2008;178:157-64.

17. Bogun F, Crawford T, Reich S, et al. Radiofrequency ablation of frequent, idiopathic premature ventricular complexes: comparison with a control group without intervention. Heart Rhythm 2007;7:863-7.

18. Echt DS, Liebson PR, Mitchell LB, et al. Mortality and morbidity in patients receiving encainide, flecainide, or placebo. The Cardiac Arrhythmia Suppression Trial. N Engl J Med 1991;324:781-8. 